\title{
APOYANDO LA INVESTIGACIÓN: NUEVOS ROLES EN EL SERVICIO DE BIBLIOTECAS DE LA UNIVERSIDAD DE NAVARRA
}

\author{
Supporting research: new roles in the library \\ services of University of Navarra
}

Isabel Iribarren-Maestro, Teresa Grandal, María Alecha, Ana Nieva y Teresa San-Julián

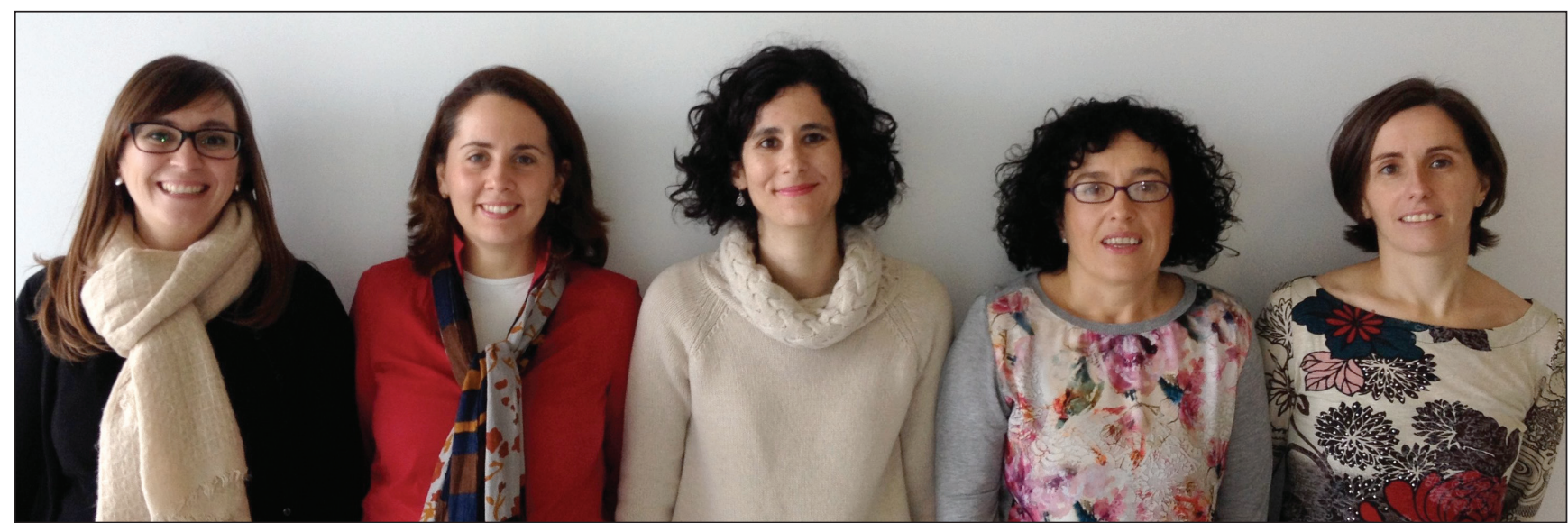

Isabel Iribarren-Maestro es doctora en documentación por la Universidad Carlos III de Madrid (UC3M) y trabaja en la Biblioteca de la Universidad de Navarra desde 2010, de la que es subdirectora desde 2012. Sus líneas de trabajo se centran en las áreas de Atención a Usuarios, especialmente el apoyo a la actividad investigadora, y de Evaluación de la Biblioteca y ha sido una de las responsables de la reciente creación del Servicio de Bibliometría. Anteriormente trabajó en el Departamento de Biblioteconomía y Documentación de la UC3M, con la que mantiene vinculación a través del Laboratorio de Estudios Métricos de Información (LEMI).

http://orcid.org/0000-0001-9264-7271

iiribarrenm@unav.es

Teresa Grandal, licenciada en humanidades por la Universidad de Navarra y máster en bibliotecas digitales por la Universidad de Valladolid, trabaja en el Servicio de Bibliotecas de la Universidad de Navarra desde hace siete años, donde gestiona el acceso y mantenimiento de los recursos electrónicos. Es bibliotecaria temática de psicología. Coordina la labor de los bibliotecarios en el proyecto de científicacvn.

https://orcid.org/0000-0001-8086-3989

tgrandal@unav.es

María Alecha es diplomada en biblioteconomía y documentación por la Universidad de Zaragoza. Desde 2008 trabaja en el Servicio de Bibliotecas de la Universidad de Navarra, donde ha desempeñado tareas en el departamento de Publicaciones Periódicas. Compagina la gestión de la Biblioteca de Arquitectura, con las labores de bibliotecaria temática de dicha área y con el asesoramiento a profesores sobre los procesos de evaluación de las agencias (Aneca/Cneai).

https://orcid.org/0000-0003-1540-1870

alecha@unav.es

Ana Nieva es licenciada en filología inglesa por la Universidad de Salamanca y trabaja en el Servicio de Bibliotecas de la Universidad de Navarra desde 2009, donde desempeña tareas como atención a usuarios (información bibliográfica y préstamo), préstamo interbibliotecario y bibliotecaria temática de ciencias. Desde 2013 coordina las tareas de los bibliotecarios en el proyecto de científicacun.

https://orcid.org/0000-0002-7773-6409

anlopez@unav.es 
Teresa San-Julián es licenciada en geografía e historia por la Universidad de Navarra y máster en biblioteconomía por la Universidad del País Vasco. Desde 2001 trabaja en el Servicio de Bibliotecas de la Universidad de Navarra, en el departamento de Catalogación en tareas relacionadas con la gestión de la colección y la catalogación y carga de recursos electrónicos. http://orcid.org/0000-0003-3736-4521

tsanjulian@unav.es

Universidad de Navarra, Servicio de Bibliotecas Campus Universitario. 31009 Pamplona, España

\section{Resumen}

La Biblioteca de la Universidad de Navarra ha ido asumiendo en los últimos años nuevos roles de apoyo a la investigación. Junto con tareas como formación a la carta o el fomento del acceso abierto a través del repositorio, se han iniciado asesoramientos a profesores en procesos de acreditación y solicitud de sexenios, así como colaboración con el sistema de gestión de datos de investigación en tareas de revisión y validación de la producción científica. Se detallan las nuevas funciones y tareas asumidas, su incidencia frente a las funciones tradicionales, las ventajas que han comportado y las líneas de futuro.

\section{Palabras clave}

Bibliotecas universitarias; Servicios bibliotecarios; Apoyo a la investigación; Publicaciones científicas; Bibliometría; Servicios de bibliometría; Profesión; Bibliotecarios temáticos; Bibliotecarios especialistas.

\section{Abstract}

In the last few years, the Library of the University of Navarra has taken on new librarian roles, mainly related to research support. Along with tasks such as on-demand training sessions and the promotion of open access to the Library's repository, we have started several new lines of research support. These primarily focus on advising professors about accreditation processes and six-year research evaluations, and providing data review and item validation support for the institutional research management system. We analyze these new roles and tasks that have been undertaken, together with their impact on traditional tasks, their benefits, and new perspectives for the future.

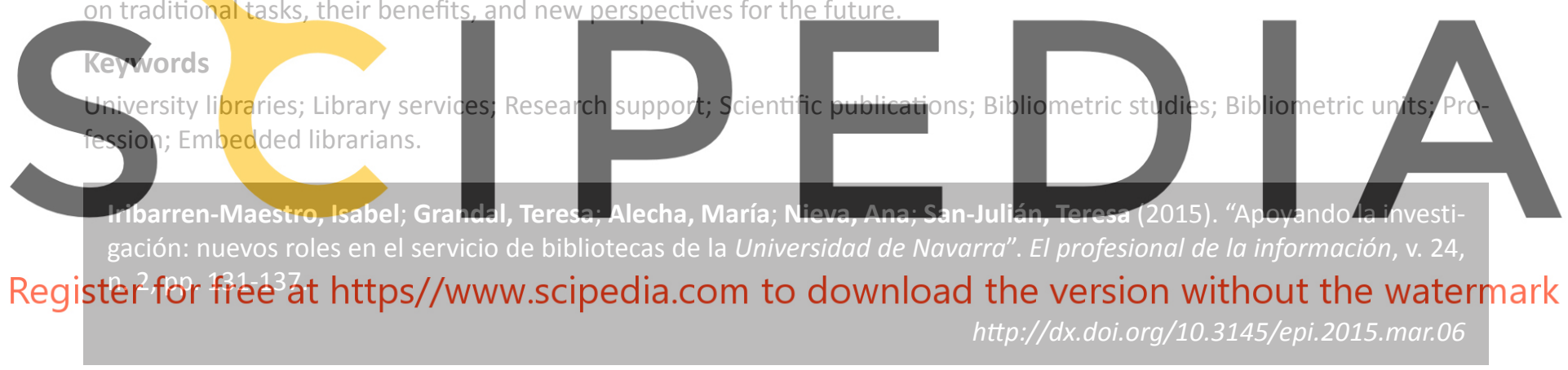

\section{Introducción}

En las últimas décadas se han producido cambios en las tareas y roles de las bibliotecas universitarias que han provocado una recreación de las mismas (Guallar, 2010). En manuales de referencia de la profesión (Area-Moreira; Hernández; Sancho, 2007; Alire; Evans, 2010, etc.) se han definido nuevas funciones como alfabetización informacional, bibliotecas digitales y preservación de documentos electrónicos. Las funciones tradicionales se ven relegadas por la agilidad que ofrece la tecnología y por el surgimiento de nuevas tareas que se adaptan mejor a las necesidades de información de los colectivos que utilizan la biblioteca. La presencia del bibliotecario como un agente activo en el proceso enseñanza-aprendizaje del alumno o en los grupos de investigación -el ya consolidado papel de "bibliotecario temático"-, ha transformado el valor de estos profesionales hasta el punto de ser sumamente necesarios como apoyo para las actividades docentes e investigadoras.

En esta línea, Anglada (2012) menciona los servicios a los investigadores como una de las principales tendencias de innovación de la biblioteca universitaria (junto con los re- positorios y el acceso abierto, los espacios renovados, las herramientas de descubrimiento y el libro electrónico), aunque su planteamiento no llega a augurar los niveles que esta actividad conlleva hoy en las bibliotecas universitarias, sólo unos pocos años después de realizarlo. El autor aborda estos servicios desde dos perspectivas: la conservación de los datos de investigación y la incorporación de los bibliotecarios en los procesos de investigación. Sin embargo, no menciona otras tareas como la gestión de los datos de producción de los investigadores, el asesoramiento en procesos de evaluación, o la obtención de indicadores bibliométricos como apoyo al gobierno de la institución.

\section{El bibliotecario se ha transformado en un agente necesario como apoyo para las ac- tividades docentes e investigadoras}

Por otro lado, Blanco y Casaldàliga (2012) focalizan la importancia del soporte a la actividad investigadora en la difusión de la producción científica, englobando bajo esta definición cuatro líneas de trabajo: 
- repositorios y política de acceso abierto;

- Portal de Producción Científica $(P P C)^{1}$;

- normalización del nombre y filiación de los investigadores;

- difusión de índices y herramientas para evaluar la actividad investigadora.

Centrándonos en las tareas de apoyo a la investigación, los profesores encuentran fundamental la asistencia que da la biblioteca en procesos de acreditación, sexenios de investigación, solicitud de proyectos, etc. Es habitual encontrar en convocatorias oficiales información que para ellos es desconocida, como menciones a bases de datos bibliográficas, fuentes de información sobre calidad de revistas, indicadores como el factor de impacto o el índice hy cada vez medidas más complejas: promedio de citas por año, porcentaje de publicaciones en el primer cuartil
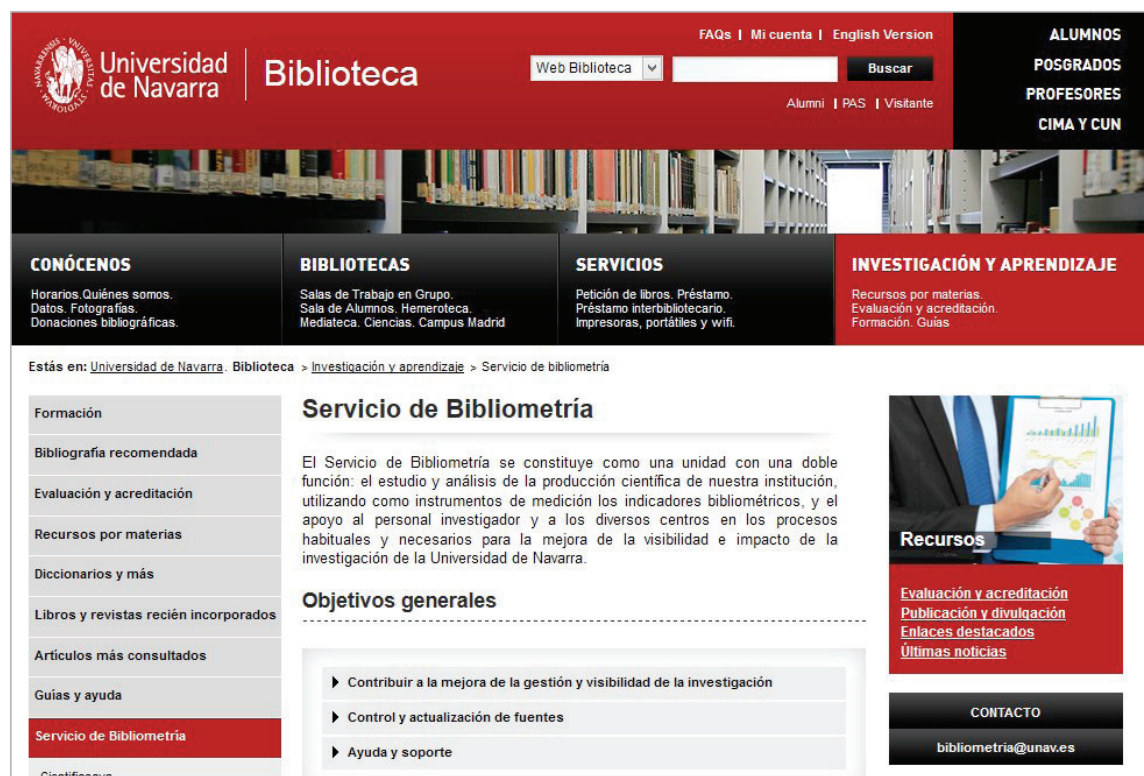
o el impacto normalizado (Españ

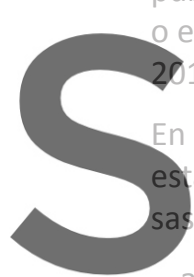
13; 2014; Aneca, 2008)

En los últimos años las bibliot

án dando respuesta a estas

formas:

través de guías o tutoriales:
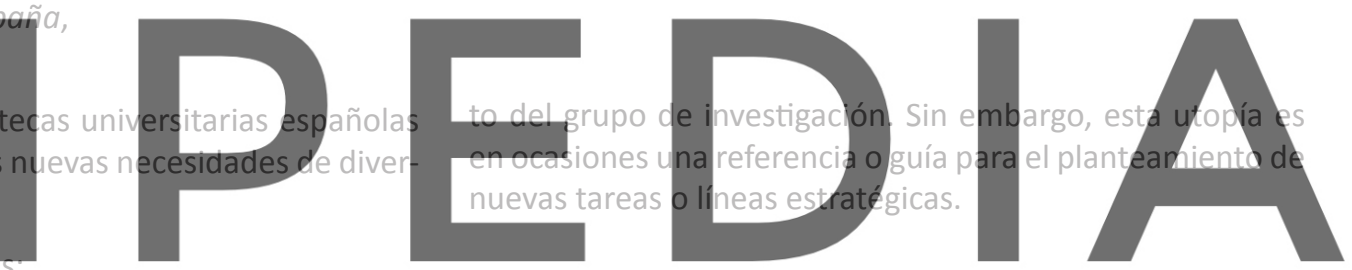

ofreciendo asistencia a procesos de acreditación o cola- S Se cuenta con bibliotecarios formados

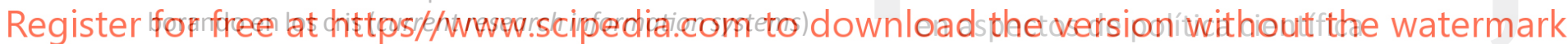
al validar registros;

las más implicadas, asumiendo tareas enfocadas no sólo al investigador sino también a la institución en su conjunto a través de un Servicio de Bibliometría o similar.

\section{El Servicio de Bibliotecas de la Universidad de Navarra (Unav)}

Este Servicio está incorporando paulatinamente desde hace unos años estas tendencias a su actividad diaria.

El origen de la adaptación de las funciones de los bibliotecarios de la Unav a las nuevas necesidades de los usuarios, y concretamente de los investigadores, se puede datar en 2009, con el inicio del proyecto de bibliotecarios temáticos. El nuevo perfil -también conocido como embebido o integrado- se ocupa de la formación en competencias informacionales, participa en las reuniones departamentales y apoya a los investigadores (González-Fernández-Villavicencio, 2012).

Torres-Salinas (2011) plasma desde un punto de vista conceptual lo que debe realizar este profesional en su apoyo a la investigación. Se trata de un planteamiento casi utópico por el elevado coste que implicaría llevarlo a cabo. Engloba desde la intervención en todo el proceso de publicación de artículos hasta tareas de gestión de la visibilidad y el impac-

En la Unav el personal del Servicio ha ido asumiendo tareas con cautela y minuciosidad, y los profesores-investigadores las han ido conociendo y reconociendo gradualmente. Aunque en principio se basaban en acciones tradicionales, como el desarrollo de la colección o el servicio de referencia, pronto fueron llevando a cabo cometidos más novedosos. En la actualidad todos los bibliotecarios temáticos de la institución (un total de 20):

- revisan y aseguran la disponibilidad de la bibliografía recomendada de las asignaturas de sus respectivas áreas;

- controlan los presupuestos de revistas;

- imparten sesiones de formación a todos los niveles (alumnos de $1^{r}$ de grado, sesiones preparatorias para la elaboración de trabajos fin de grado y fin de master, en programas de posgrado, y profesores);

- elaboran guías temáticas con el programa SubjectsPlus²;

- validan los items de producción científica incluidos en la base de datos de gestión de la investigación de la Universidad, científicacvn, que explicaremos más adelante.

Todo ello siguiendo un plan de formación en el extranjero, basado en estancias de tres meses, que la gran mayoría ha desarrollado en bibliotecas de referencia de Estados Unidos 
o Inglaterra (Sanz-Santacruz; Serrano-Vicente; Iturbide-Tellechea, 2013). Como se puede observar ante esta relación de tareas, algunas de ellas se perfilan claramente como acciones de apoyo a la investigación.

Coincidiendo prácticamente en el tiempo, el Servicio de Bibliotecas puso en marcha también el repositorio institucional Dadun (Depósito académico digital UN) (SerranoVicente; Cózar-Santiago; Iturbide-Tellechea, 2012). Este compromiso por el acceso abierto es patente en el volumen actual de Dadun: 14.235 documentos en Depósito académico y 16.451 en Revistas $U N^{3}$.

Otra tarea que se viene llevando a cabo desde 2010 es el apoyo a procesos de acreditación y sexenios. Esta labor se inició sin ser un servicio ofertado como tal, y se realizaba en entrevistas personales con los profesores. Sin embargo, la creciente y rápida demanda propició la elaboración de una guía de recursos fundamentales para los investigadores ${ }^{4}$ y la formación de algunos bibliotecarios en aspectos de política científica. La firma de un convenio en 2011 para solicitar sexenios entre la Unav-que por ser una universidad privada carecía de este privilegio- y la Comisión Nacional de Evaluación de la Actividad Investigadora (Cneai), terminó por afianzar el servicio, que actualmente está muy bien valorado por los profesores y por el Vicerrectorado de Investigación.

La última labor de gran envergadura que los bibliotecarios

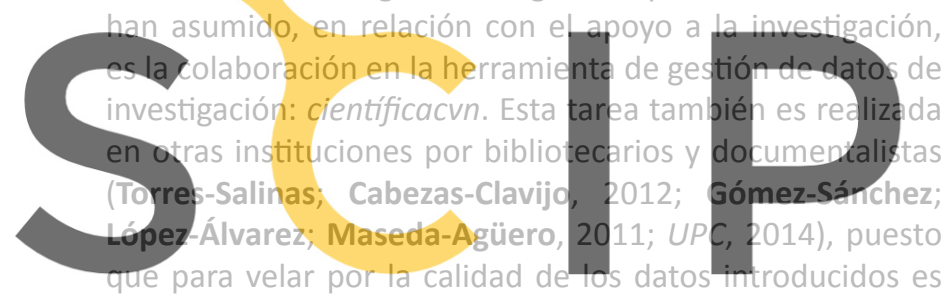

necesaria su revisión (Fernández-Fernández, 2014)

Register for free at https//www.scipedia.com to

\section{La validación de items migrados o de \\ nueva creación en científicacvn se ha consolidado como una actividad más de los bibliotecarios temáticos}

Científicacvn está activa en la Unav desde principios de 2013 (Torres-Salinas, 2013), aunque no es la primera aplicación de este tipo que tiene la Universidad, ya que desde 2006 los investigadores contaban con Universitas XXI, creada por la Oficina de Cooperación Universitaria (OCU). Después de unos años de trabajo con ella se optó por elaborar una aplicación basada en el estándar CVN (curriculum vitae normalizado) por las ventajas de intercambio que este estándar $\mathrm{XML}$ ofrece, ya que posibilita que los modelos de currículum de las instituciones que lo utilizan sean interoperables entre sí (Báez et al., 2008).

En el arranque de científicacvn se migraron los datos de producción científica consignados en Universitas XXI durante su vigencia en la Unav. Debido a que el control de los datos había recaído en los propios investigadores, no estaban revisados y contaban con múltiples errores. Por esta razón fue necesaria una ardua tarea de corrección por parte de los bibliotecarios, y hasta la fecha se han validado 11.599 registros. La tarea de revisión se ha consolidado como una actividad más de los bibliotecarios temáticos, en los registros de nueva creación, cuando se trata de publicaciones en revistas, libros y capítulos de libro.

Además, desde la Biblioteca se han asumido también otras funciones, como la formación de investigadores en el uso de científicacvn, la corrección del tipo de ítem -cuando se den errores de adscripción- o el mantenimiento de la masterlist de revistas.

\section{Líneas de futuro: el nuevo Servicio de Bibliometría}

En paralelo a lo descrito sobre el Servicio de Bibliotecas, la Unav cuenta con un técnico experto en evaluación científica que desde hace años se está encargando de la evaluación de la investigación -a través de la aplicación_Científica (TorresSalinas, 2008)-, el asesoramiento para la presentación a convocatorias o la memoria de investigación de la institución.

Desde el Vicerrectorado de Investigación se ha visto la necesidad de unificar todas estas competencias de apoyo a la investigación en una única entidad, dando como resultado la creación del nuevo Servicio de Bibliometría, en el que se integran las tareas de evaluación científica y las de apoyo a la investigación descritas anteriormente. La interdiscipli-

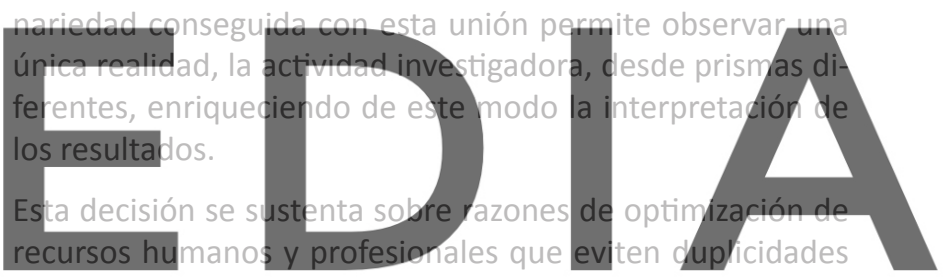

en las tareas, así como de despersonalización y diversificación de cometidas. De esta manera.se pretende que el nuedown 18 ad the versign without the watermark

el investigador, y pueda ofrecer una carta de servicios más extensa, capaz de abordar proyectos de mayor envergadura.

El nuevo Servicio de Bibliometría se presenta dotado de cierta autonomía, articulado en parte por el Servicio de Bibliotecas, pero con dependencia directa del Vicerrectorado de Investigación. Nace con una plantilla compuesta por cinco bibliotecarias -todas con dedicación parcial- y el técnico de investigación, y en su carta inicial de servicios sigue la línea de propuestas teóricas de otras unidades de bibliometría (Blanco; Casaldàliga, 2012; Torres-Salinas; Jiménez-Contreras, 2012; Torres-Salinas; Cabezas-Clavijo, 2012).

Ofrece cobertura en tres aspectos:

- control de las fuentes de información sobre investigación, fundamentalmente, científicacvn: formación y soporte a los investigadores, alimentación automática del sistema, nuevos desarrollos y mejoras, cuadros de mando, etc.;

generación de informes de análisis, prospectiva y vigilancia: realización de memorias de investigación, elaboración de informes, apoyo a la presentación de convocatorias, divulgación de resultados, etc.;

- formación, asesoramiento y consulta experta: formación de investigadores en temas de indicadores y evaluación, acreditación y solicitud de sexenios, asesoría a centros, etc. 
En la explotación de científicacun como herramienta de análisis bibliométrico principal, se busca obtener una visión holística de la actividad académica e investigadora generada en la Universidad que permita analizarla en sus cinco vertientes: básica, aplicada, tecnológica, educativa y divulgativa. Además, esta aplicación viene a dar solución a las tradicionales limitaciones del uso de las bases de datos bibliográficas como fuente bibliométrica (sólo recogen las publicaciones consignadas en determinadas fuentes), y gracias al estándar CVN, subsana también la falta de homogeneidad detectada en las fuentes de datos institucionales. (De-Filippo et al., 2011; Sanz-Casado; Lascurain; IribarrenMaestro, 2006).

Al hilo de dicha explotación, y entroncando con las competencias del bibliotecario temático en la validación de los items de producción investigadora, se ha demostrado que existe ya una simbiosis entre investigadores y Biblioteca: el investigador se beneficia del know how del bibliotecario sobre las fuentes de información para la evaluación científica, y la Biblioteca logra una mejora en la gestión de la colección al permitirle conocer los hábitos de publicación.

\section{Conclusiones}

Las tareas de apoyo a la investigación desempeñadas por el personal bibliotecario de la Unav han tenido una clara incidencia en la variedad de tareas asumidas por la Biblioteca. forma de trabajar, han proporcionado una serie de ventajas que se muestran en la figura 1.

\section{Se dará cobertura en control de fuentes} de información, generación de informes bibliométricos y consulta experta

El futuro de este nuevo Servicio se centra en tres líneas de acción fundamentales que permitan mejorar su rendimiento y la visibilidad de sus datos:

- una vez consolidadas las tareas descritas, se orientarán los esfuerzos hacia la sincronización total entre científicacvn y el repositorio institucional Dadun, aunando de este modo dos líneas estratégicas de la Biblioteca, tal y como se hace en otras (Álvarez-de-Toledo-Saavedra, 2012; De-Castro, 2013; UPC, 2014), y desempeñando cada una el rol para el que fueron creadas (Joint, 2008);

en consonancia con el fomento de la visibilidad de la investigación, se conectará la aplicación científicacvn con identificadores de autor como Orcid o ResearcherID, o con plataformas como Researchgate;

se generarán notas divulgativas a partir de la producción de los autores para dar a conocer en distintos medios los últimos avances de investigación de la Unav.
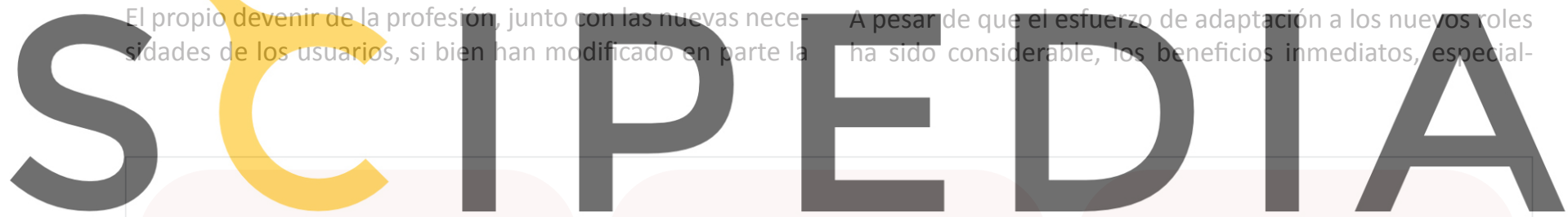

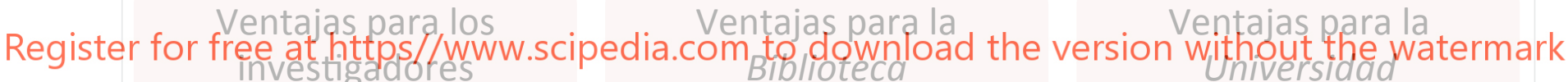

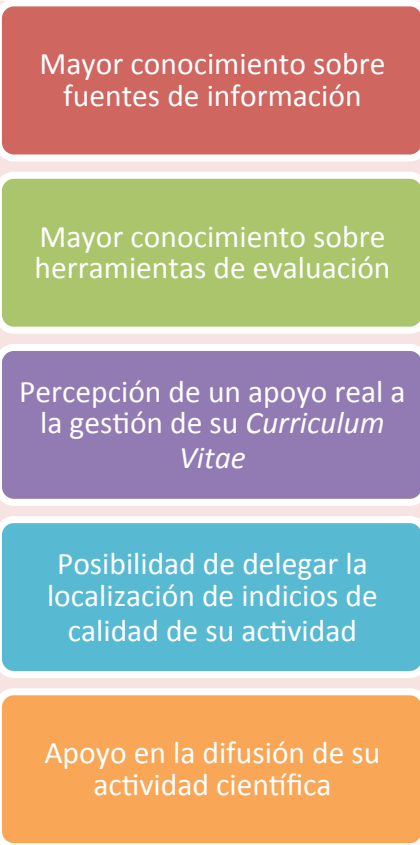

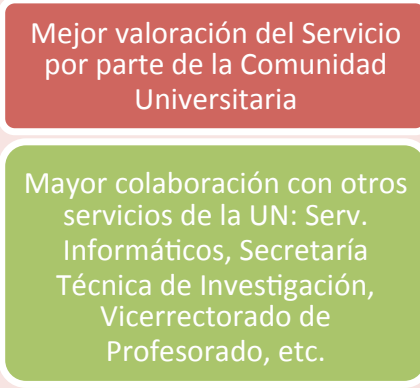

Asunción de nuevas tareas dentro de la universidad:

Formación y atención a los

investigadores en el uso de Científicacvn

Mejora en la gestión de la colección gracias al

conocimiento de las fuentes

habituales de publicación y

consumo de información por

parte de investigadores

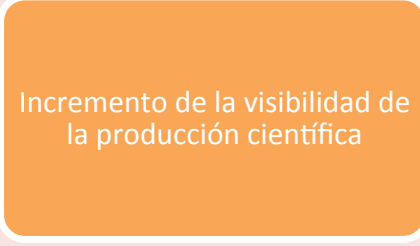

Mejores resultados en

procesos de acreditación,

solicitud de sexenios,

convocatorias, etc.

Mayor cobertura en la

obtención de datos

fundamentales para el

gobierno de la investigación

Figura 1. Ventajas observadas de la realización de tareas de apoyo a la investigación 
mente en términos de percepción y valoración de nuestros usuarios, han merecido la pena.

Afrontamos con grandes expectativas la puesta en marcha del nuevo Servicio de Bibliometría, que enriquecerá y desarrollará nuestra actividad como profesionales bibliotecarios. Si a todo ello le sumamos un análisis en términos de sostenibilidad, según las variables de coste, uso y percepción propuestas recientemente en la literatura (Anglada, 2014), parece razonable augurarle un largo futuro.

La simbiosis entre investigadores y bibliotecarios es ya una realidad

\section{Notas}

1. PPC es el sistema de gestión de datos de investigación de la UPC. Este tipo de programa es conocido como cris (current research information system).

2. SubjectsPlus es una aplicación de código abierto desarrollada por Ithaca College, que se utiliza para elaborar guías temáticas de recursos de información en formato web. http://www.subjectsplus.com

3. Datos obtenidos el 17/10/2014.

4. hittp://apps-bibl.si.unav.es/sp/subjects/guide.php?subject=apoyo_

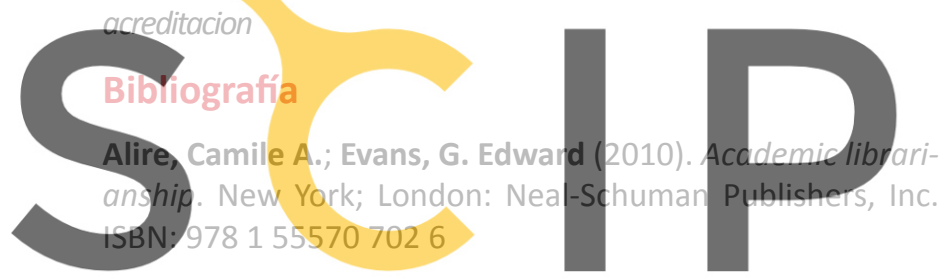

Álvarez-de-Toledo-Saavedra, Luisa (2012). "Control biblio-

Registáficovédifusión de la producgión científica de la Universipp. 6396-642.

hitp://dx.doi.org/10.3145/epi.2012.nov.12

Aneca (2008). Programa Academia: Principios y orientaciones para la aplicación de los criterios de evaluación. V. 2.0. 31/01/2008.

http://www.aneca.es/Programas/ACADEMIA/ACADEMIAinstrucciones-para-la-solicitud/Documentos-del-Programa

Anglada, Lluís (2012). "Bibliotecas universitarias: cabalgando la tecnología, siguiendo al usuario". El profesional de la información, v. 21, n. 6, pp. 553-556.

http://www.elprofesionaldelainformacion.com/contenidos/2012/ noviembre/01.pdf

http://dx.doi.org/10.3145/epi.2012.nov.01

Anglada, Lluís (2014). "Are libraries sustainable in a world of free, networked, digital information?". El profesional de la información, v. 23, n. 6, pp. 603-611.

http://www.elprofesionaldelainformacion.com/contenidos/2014/ nov/07_esp.pdf

http://dx.doi.org/10.3145/epi.2014.nov.07

Area-Moreira, Manuel; Hernández, Fernando; Sancho, Juana-María (2007). De la biblioteca al centro de recursos para el aprendizaje y la investigación. Barcelona: Octaedro. ISBN: 9788480639095
Báez, José-Manuel; Peset, Fernanda; Núñez, Florencio; Ferrer, Antonia (2008). "CVN: normalización de los currículos científicos". El profesional de la información, v. 17, n. 2, pp. 213-220.

http://eprints.rclis.org/12244

http://dx.doi.org/10.3145/epi.2008.mar.12

Blanco, Elena; Casaldàliga, Anna (2012). "El papel de la biblioteca en la difusión de la producción científica en la Universitat Pompeu Fabra". El profesional de la información, v. 21, n. 6, pp. 627-631.

http://repositori.upf.edu/handle/10230/20055

http://dx.doi.org/10.3145/epi.2012.nov.10

De-Castro, Pablo (2013). "La integración de cris y repositorio: sistematizando el acceso abierto". En: Semana internacional del acceso abierto.

http://es.slideshare.net/socialbiblio/integracioncrisrepositorio-sistematizando-el-acceso-abierto

De-Filippo, Daniela; Sanz-Casado, Elías; Ubano-Salido, Cristóbal; Ardanuy, Jordi; Gómez-Caridad, Isabel (2011). "El papel de las bases de datos institucionales en el análisis de la actividad científica de las universidades". Revista española de documentación científica, abril-junio, v. 34, n. 2, pp. 165189.

http://dx.doi.org/10.3989/redc.2011.2.797

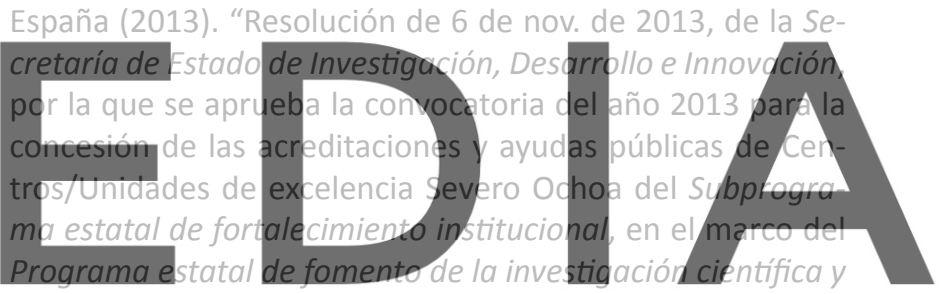
técnica de excelencia del Plan estatal de investigación cientí-

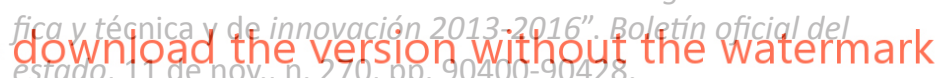
http://www.boe.es/diario_boe/txt.php?id=BOE-A-2013-11792

España (2014). "Resolución de 6 de agosto de 2014, de la Secretaría de Estado de Investigación, Desarrollo e Innovación, por la que se aprueba la convocatoria para el año 2014 del procedimiento de concesión de ayudas correspondientes al Programa estatal de investigación, desarrollo e innovación orientada a los retos de la sociedad, en el marco del Plan estatal de investigación científica y técnica y de innovación 2013-2016". Boletín oficial del estado, 8 de agosto, n. 192, pp. 63854-63899.

http://www.boe.es/diario boe/txt.php?id=BOE-A-2014-8602

Fernández-Fernández, Rocío (2014). “Biblioteca, CVN y CRIS: La gestión del currículum del investigador en la Universidad de Burgos". En: VI Jornadas Bucle sobre bibliotecas universitarias, redes para la cooperación.

http://es.slideshare.net/BucleJornadas/cris-cvn-ocuuniversitas-xxi-jornadas-bucle

Gómez-Sánchez, Alicia-Fátima; López-Álvarez, Victoria; Maseda-Agüero, Irene (2011). "Servicios bibliométricos en las bibliotecas especializadas: utilidad y aprovechamiento de una base de datos de producción científica". En: Bibliosalud 2011: XIV Jornadas nacionales de información y documentación en ciencias de la salud. Sevilla: Biblioteca Virtual 
del Sistema Sanitario Público de Andalucía, pp. 95-98. ISBN: 9788461495221

http://eprints.rclis.org/15934

González-Fernández-Villavicencio, Nieves (2012). "Servicios de referencia en bibliotecas universitarias: tendencias y plan de marketing". El profesional de la información, v. 21, n. 6, pp. 567-576.

http://eprints.rclis.org/18133/1/03-Gonza\%CC\%81/ezFerna\%CC\%81ndez-Villavicencio.pdf http://dx.doi.org/10.3145/epi.2012.nov.03

Guallar, Javier (2010). "Reflexiones sobre bibliotecas. Entrevista a Lluís Anglada". El profesional de la información, v. 19, n. 5, pp. 545-551.

http://www.elprofesionaldelainformacion.com/contenidos/2010/ septiembre/entrevista_anglada.pdf

http://dx.doi.org/10.3145/epi.2010.sep.16

Joint, Nicholas (2008). "Current research information systems, open access repositories and libraries: Antaeus". Library review, v. 57, n. 8, pp. 570-575

http://dx.doi.org/10.1108/00242530810899559

Sanz-Casado, Elías; Lascurain, María-Luisa; Iribarren-Maestro, Isabel (2006). "Luces y sombras en la evaluación de la investigación en ciencias sociales y humanidades”. En: Ibarra, Andoni; Castro, Javier; Barrenechea, J. La evaluación de la actividad científica en ciencias sociales y humanidades
Tellechea, María-Arántzazu (2012). “Desarrollo de la introducción de contenidos en Dadun: diversidad de métodos y flexibilidad de sistemas". En: Quintas jornadas Os-repositorios. La motricidad de los repositorios de acceso abierto. http://dadun.unav.edu/handle/10171/22845

Torres-Salinas, Daniel (2008). “_Científica: una aplicación informática para medir la ciencia, una aplicación para la toma decisiones". En: VIII Semana de la ciencia. Investigación cuantitativa de la actividad científica, Madrid, 12 de noviembre.

http://es.slideshare.net/torressalinas/cientifica-presentation

Torres-Salinas, Daniel (2011). "Integrados en la investigación: los embedded librarians". Anuario ThinkEPI, v. 5, pp. 48-51.

Torres-Salinas, Daniel (2013). Científicacvn: Nuevo sistema de información científica de la Universidad de Navarra. Sesión de presentación. Universidad de Navarra, 25 y 26 de Marzo de 2013

http://www.slideshare.net/torressalinas/cientificacun

Torres-Salinas, Daniel; Cabezas-Clavijo, Álvaro (2012). “Herramientas para la evaluación de la ciencia en universidades y centros I+D: descripción y usos". Anuario ThinkEPI, v. 6, pp. 142-146.

http://digibug.ugr.es/handle/10481/20220\#. VNBbwmRWUUW
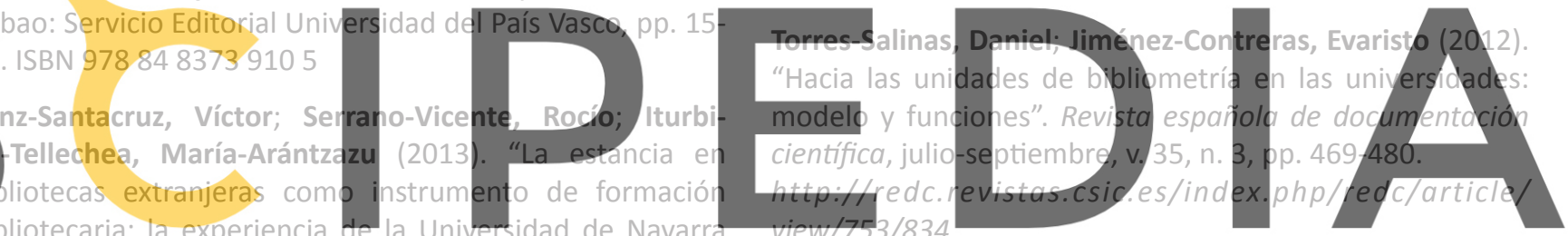

(2003-2010)". Revista española de documentación científi-

http://dx.doi.org/10.3989/redc.2012.3.959

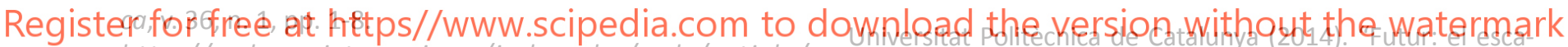
http://redc.revistas.csic.es/index.php/redc/article/ view/783/911

Serrano-Vicente, Rocío; Cózar-Santiago, Amparo; Iturbide-

parate de la producción científica de la UPC". Sala de premsa http://www.upc.edu/saladepremsa/al-dia/mes-noticies/

futur-el-escaparate-de-la-produccion-cientifica-de-la-upc

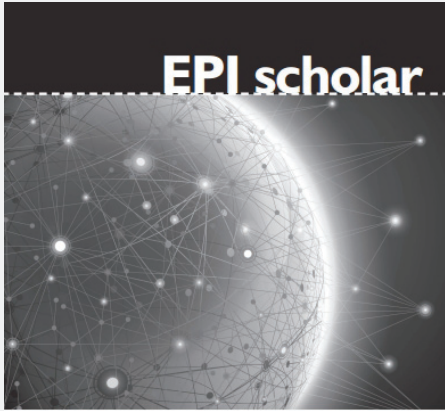

CIBERMETRÍA MIDIENDO EL ESPACIO RED ENRIQUE ORDUNA-MALEA ISIDRO F. AGUILLO

\section{Nueva colección EPI Scholar}

\section{Libros académicos y científicos de Información y Documentación}

\section{Primer título: \\ Cibermetría. Midiendo el espacio red de Enrique Orduña-Malea e Isidro F. Aguillo}

A pesar del crecimiento de la disciplina de la Cibermetría desde mediados de la década de los noventa, son escasos los libros académicos o manuales dedicados en exclusiva a la misma desde un contexto de las ciencias de la información y documentación. Este libro pretende cubrir este claro hueco en la literatura tanto nacional como internacional.

http://www.elprofesionaldelainformacion.com 